\title{
COMPRENDRE LA MONDIALISATION
}

(CYCLE 1)

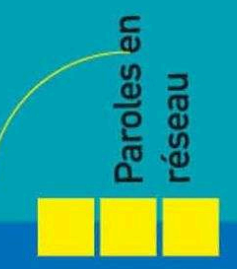

Actes des rencontres du 16 janvier au 6 mars 2006

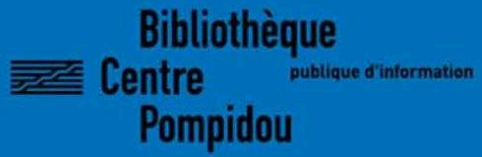




\title{
Comprendre la mondialisation I
}

\author{
Brice Couturier, Lionel Fontagné, Philippe Manière, Philippe Martin et \\ Bernard Nadoulek
}

DOI : 10.4000/books.bibpompidou. 1275

Éditeur : Éditions de la Bibliothèque publique d'information Année d'édition : 2008

Date de mise en ligne : 17 janvier 2014

Collection : Paroles en réseau

ISBN électronique : 9782842462147

\section{Sbooks}

http://books.openedition.org

\section{Édition imprimée}

ISBN : 9782842461126

Nombre de pages : 32

Référence électronique

COUTURIER, Brice; et al. Comprendre la mondialisation I. Nouvelle édition [en ligne]. Paris : Éditions de la Bibliothèque publique d'information, 2008 (généré le 02 février 2021). Disponible sur Internet: <http://books.openedition.org/bibpompidou/1275>. ISBN : 9782842462147. DOI : https://doi.org/ 10.4000/books.bibpompidou.1275.

(c) Éditions de la Bibliothèque publique d'information, 2008 Conditions d'utilisation :

http://www.openedition.org/6540 


\title{
Comprendre la mondialisation (cycle 1)
}

\author{
Manifestation organisée par le service Animation \\ de la Bibliothèque publique d'information \\ (pôle Action culturelle et Communication). \\ Cycle de conférences et de débats : janvier/mars 2006, \\ dans la Petite Salle du Centre Pompidou.
}

Bibliothèque

在 Centre

Pompidou

publisue findarmatioe

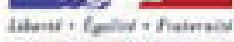

Rircaugue Funcuse

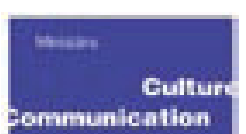




\author{
Président \\ du Centre Pompidou \\ Alain Seban \\ Directrice générale \\ du Centre Pompidou \\ Agnès Saal \\ Directeur de la Bpi \\ Thierry Grognet \\ Responsable du pôle \\ Action culturelle \\ et communication \\ Philippe Charrier \\ Chef du service \\ Animation \\ Emmanuèle Payen \\ Responsable \\ Édition/Diffusion \\ Arielle Rousselle \\ Conférences \\ et débats \\ Conception et \\ Organisation \\ Francine Figuière \\ Publication \\ Chargées d'édition \\ Coralie Salmeron \\ Virginie Gazil \\ Chrystel Vannier \\ Mise en page \\ Fabienne Charraire \\ Virginie Gazil \\ Chrystel Vannier \\ Note à l'usage des internautes: \\ Cette édition a été enrichie de liens vers des sites, \\ rendant possible un élargissement \\ et un approfondissement des sujets abordés. \\ Or, Internet étant un outil vivant et en constante \\ évolution, il est possible que certains liens créés ne \\ soient plus valides au moment où vous consulterez \\ ce dossier. \\ Par ailleurs, vous pouvez écouter les interventions \\ sur la base d'archives sonores de la Bpi : \\ http://archives-sonores.bpi.fr /
}

Catalogue disponible sur

http: //www.bpi.fr, rubrique Publication ou

http: //www.editionsdelabibliothèqueabpi.com

(c) Éditions de la Bibliothèque publique d'information/

Centre Pompidou, 2008.

ISBN 978-2-84246-112-6

ISSN 1765-2782 


\section{Sommaire}

Lundi 16 janvier 2006

Qu'est-ce que la mondialisation?

Ouverture (non disponible)

Érik Le Boucher

Mondialisation et civilisations

Bernard Nadoulek

Les faces multiples

de la mondialisation contemporaine (à venir)

Olivier Mongin

La nouvelle économie-monde (à venir)

Daniel Cohen

Débat (non disponible)

Modérateur : Éric Le Boucher

Lundi 30 janvier 2006

La mondialisation économique :

chance ou fléau pour les pays pauvres?

Ouverture

Brice Couturier

Commerce, ouverture, pauvreté

Lionel Fontagné

Commerce et lutte contre la pauvreté :

une équation à plusieurs inconnues (non disponible)

Céline Trublin

Bâtir un secteur financier ouvert à tous (non disponible)

Maria Nowak

Le paradoxe de la mondialisation en Afrique là venir)

Stephen Smith

Débat (non disponible)

Modérateur: Brice Couturier 


\section{Sommaire}

\section{Lundi 20 février 2006}

\section{Les pays riches dans une économie mondialisée}

\section{Ouverture}

Philipe Manière

Compétitivité mondiale, cohésion sociale

et modèle social européen sont-ils compatibles? (non disponible) Joël Décaillon

Mondialisation et territoires

Philippe Martin

Pourquoi les Français se méfient

du capitalisme financier (non disponible)

David Thesmar

Vers une mondialisation socialement responsable (à venir)

Thierry Verdier

Débat (non disponible)

Modérateur : Philippe Manière

\section{Lundi 6 mars 2006}

\section{La mondialisation économique peut-elle se réguler?}

Ouverture (non disponible)

Érik Izraelewicz

Comment gouverner la mondialisation? (non disponible)

Pierre Jacquet

Réformer les institutions internationales et les instruments pour mieux réguler la finance globalisée (non disponible)

Dominique Plihon

Globalisation financière et rôles

du Fonds monétaire international (à venir)

Hélène Rey

Gouvernance mondiale, gouvernance régionale :

quelles pistes pour les pays en développement? (non disponible)

Akiko Suwa-Eisenmann

Débat (non disponible)

Modérateur : Érik Izraelewicz 


\section{Lundi 16 janvier 2006}

\section{Qu'est-ce que la mondialisation?}

Ouverture (non disponible)

Érik Le Boucher

Mondialisation et civilisations

Bernard Nadoulek

Les faces multiples

de la mondialisation contemporaine (à venir)

Olivier Mongin

La nouvelle économie-monde là venir)

Daniel Cohen

Débat (non disponible)

Modérateur : Éric Le Boucher 


\section{Mondialisation et civilisations}

\section{Bernard Nadoulek*}

Mon intervention étant limitée, j'essaierai d'esquisser une brève analyse des rapports entre mondialisation et civilisations pour vous apporter quelques pistes de réflexion sur la contradiction entre le caractère universel de la globalisation et les secousses identitaires qui la perturbent. Après une introduction, je traiterai ce sujet en trois parties et une conclusion. L'introduction nous permettra de détailler le paradoxe d'un mouvement de mondialisation que nous considérons comme "global ", alors même qu'il est tissé de revendications identitaires. Dans la première partie, nous aborderons une illusion ethnocentriste occidentale, qui ne s'est pas encore dissipée, et qui consiste à croire que la démocratie libérale est devenue un concept universel porté par la mondialisation. Et ce, au moment même où il est rejeté par la plupart des pays non occidentaux. Dans la deuxième partie, nous verrons quau rebours de cette illusion, le dirigisme asiatique, dont la réussite flagrante porte la croissance des pays émergents, n'est fondé ni sur la démocratie, ni sur l'économie libérale, au sens où l'on entend ces deux concepts en Occident. Dans la troisième partie, nous verrons comment l'intégrisme islamique incarne lui aussi un refus du discours de l'Occident. Bien qu'il incarne un échec, contrairement à la réussite du modèle asiatique, on ne peut sous-estimer la capacité de nuisance de l'intégrisme qui, lui aussi, se présente comme un universalisme dans une période de retour en force mondiale du religieux. Enfin, la conclusion nous permettra d'entrevoir que dans la crise systémique mondiale qui s'annonce, si nous souhaitons limiter les effets destructeurs des bouleversements qui s'annoncent, grâce à des négociations internationales, nous ne pouvons plus évacuer les différences culturelles.

\section{Introduction}

Mondialisation et civilisation, deux termes presque contradictoires.

La mondialisation, le phénomène de décloisonnement économique, technologique et politique auquel nous assistons depuis les années 1980: un concept synonyme de marche en avant vers l'avenir et de progrès. À l'inverse, les civilisations se caractérisent par un rapport avec l'Histoire, les traditions, les religions: un concept qui semble a priori orienté vers le passé. Pourtant cette opposition ne vaut que de manière formelle. Même si la mondialisation favorise la montée en puissance d'une culture consumériste, superficielle et globale - Microsoft, Coca Cola, McDonald's, etc. -, elle provoque aussi d'innombrables revendications identitaires: nous ne voulons plus être yougoslaves, mais serbes, croates ou bosniaques, non plus rwandais, mais hutus ou tutsis, non plus irakiens, mais chiites, sunnites ou kurdes, même si nous devons tuer ou mourir pour cela. à la fin des années 1980, l'opinion courante était que la mondialisation allait provoquer un immense processus d'uniformisation. Or aujourd'hui, nous nous apercevons que les identités culturelles n'en finissent plus de s'affirmer, aussi bien de manière positive, pour créer du lien social, que de façon négative, à travers les intégrismes. Depuis la publication du livre de Samuel Huntington ${ }^{1}$, nous pouvons avoir l'impression que « le choc des civilisations " représente l'un des signes annonciateurs du refus d'un monde global, dépersonnalisé, où chacun tomberait dans un bouillon cosmopolite uniforme. Mon hypothèse est la suivante: loin d'être un 
retour vers le passé, la montée en puissance des civilisations constitue peut-être l'une des seules possibilités, dans chaque grande aire culturelle, pour adopter des formes de capitalisme et de démocratie cohérentes avec les valeurs traditionnelles locales.

Tout commence entre les années 1970 et 1980. Avant cette période, le concept de civilisation était complètement disqualifié en tant que moteur des enjeux identitaires lors des deux premiers conflits mondiaux. En effet, les travaux sur les civilisations, de Spengler par exemple, avaient été utilisés pour justifier la montée des nationalismes qui avait accompagné les deux premières guerres mondiales. Plus encore, des années 1950 aux années 1980, la situation internationale était structurée autour des rapports Est/Ouest, de la guerre froide et de la lutte entre communisme et capitalisme. La notion de civilisation était inopérante.

Dans les années 1970 puis 1980, le concept de civilisation réémerge aux états-Unis sous la forme d'un projet de civilisation universelle qui se construit empiriquement à travers les pratiques des milieux d'affaires et l'enseignement des business schools. Pour les businessmen américains, il n'était certes pas surprenant d'être confrontés à des différences de civilisations, de langues, de traditions, de coutumes ou de religions. Pas de scoop en la matière puisque les différences de "coutumes" sont décrites depuis Hérodote au $\mathrm{VI}^{\mathrm{e}}$ siècle avant notre ère. Mais, au-delà de ces différences, il existe au moins un domaine où une civilisation quasi universelle est en train de s'affirmer, celui du business. Au moment où les business schools réinventent le management comme une science universelle de la gestion, sur les fondements objectifs de la finance et du marketing, il n'y aurait qu'une seule manière de faire des affaires: grâce aux rendements d'échelle, à la maîtrise des courbes d'expérience, au contrôle de gestion, etc., pour dégager un profit optimum. En résumé, au-delà des différences culturelles, qui semblent promises à rejoindre les musées du patrimoine de l'humanité, il y aurait au moins un domaine où tous les hommes parlent un langage commun: celui des affaires. L'idée émergeait dans le contexte d'une remontée en puissance des États-Unis qui, sous la présidence de Ronald Reagan, revenaient à un néolibéralisme virulent. Le concept de civilisation est donc revenu au centre des débats pour être en partie nié puisqu'il s'agit de l'affirmation d'une civilisation au sens universel de ce terme, mais aussi d'une négation du concept de civilisation pris dans son sens identitaire.

\section{Universalisation de la démocratie et du libéralisme?}

En 1989, avec la chute du mur de Berlin, ce point de vue va prendre une dimension philosophique et idéologique, notamment à travers un article de Francis Fukuyama sur " la fin de l'Histoire ${ }^{2}$ ». Pour Fukuyama, au cours $\mathrm{du} \mathrm{xx}^{\mathrm{e}}$ siècle, trois idéologies universelles se sont affrontées de manière récurrente: le fascisme, le communisme et la démocratie libérale. Fukuyama considère que le fascisme a été disqualifié par les deux guerres mondiales. Même si des régimes fascistes existent encore, plus personne ne pense que ce modèle représente une solution d'avenir. Avec la chute du mur de Berlin, le communisme s'écroule également. Seule la plus fragile de ces idéologies demeure, celle qui apparaissait 
comme la moins monolithique, mais qui s'est avérée la plus souple et a survécu aux convulsions à la fois militaires, politiques et économiques $\mathrm{du} \mathrm{Xx}^{\mathrm{e}}$ siècle. L'idée fondamentale de Fukuyama est que la culture occidentale est devenue, sinon universelle, du moins en voie d'universalisation puisqu'elle a perdu tout concurrent. Fukuyama admet que beaucoup de pays ne sont ni démocrates ni libéraux, mais il prédit que toutes les nations n'auront d'autre possibilité que d'adopter ces deux valeurs occidentales - le capitalisme sur le plan économique et la démocratie sur le plan politique - s'ils veulent réussir à entrer dans un cycle vertueux de développement.

Pour les pays non occidentaux, plus des deux tiers des États membres de l'onu, ce discours n'est pas innocent, il représente le nouvel impérialisme idéologique de l'Occident recouvrant ses intérêts économiques. Il est aussi celui que tiennent le FMI et la Banque mondiale, lesquels exigent une démocratisation et une ouverture des frontières économiques de tous les pays souhaitant emprunter de l'argent pour leur développement. Or, pour les élites de ces pays en voie de développement, ce discours n'a rien à voir avec un projet généreux de l'Occident qui chercherait à promouvoir les meilleures méthodes pour se développer, à partir de la démocratie et de l'économie de marché. Au contraire, il s'agit d'une tentative des pays développés pour conserver leur suprématie.

Par exemple, dans les années 1970, au moment des premier et deuxième chocs pétroliers, les banques occidentales ont vu affluer dans leurs caisses une masse de pétrodollars, argent qu'elles n'ont pas laissé dormir et ont prêté de manière massive, notamment aux pays d'Amérique latine dont une grande partie était, à cette époque, sous le joug des dictatures. De fait, l'argent qui leur a été prêté n’a pas servi au développement de ces pays mais à lutter contre le communisme, c'est-à-dire à massacrer leur propre jeunesse, ou à alimenter les comptes offshore des dictateurs comme Pinochet ou Videla. Au début des années 1980, au moment où ces pays reviennent à la démocratie, les États d'Amérique latine sont les pays les plus endettés du monde sans s'être développés pour autant. C'est alors qu'ils menacent de ne plus payer leur dette, ce qui aurait eu pour effet de faire sauter le système financier et monétaire international. Les banquiers occidentaux apprennent qu'il est parfois dangereux de prêter de l'argent à une dictature. Résultat, dans beaucoup de pays non occidentaux, on estime que si nous voulons qu'ils deviennent des régimes démocratiques, c'est tout simplement pour nous assurer de la pérennité de nos prêts: un régime démocratique étant plus légitimement responsable des aides qui lui sont accordées.

Par ailleurs, sur le plan économique, un certain nombre de dirigeants des pays en voie de développement expliquent que quand ils laissent l'agriculture occidentale, largement subventionnée et productiviste, pénétrer leurs pays, où on travaille encore la terre de manière quasi médiévale, leur agriculture s'écroule, parfois achevée par l'aide humanitaire. Il en va de même pour les industries des pays en voie de développement qui ne peuvent soutenir la concurrence de la productivité et de la compétitivité des entreprises occidentales.

Ainsi, pour les dirigeants des pays en voie de développement, quand les Occidentaux leur prêchent l'économie de marché et l'ouverture 
économique, c'est pour favoriser les intérêts de leurs multinationales. C'est ainsi que le discours sur la démocratie libérale apparait comme une prolongation idéologique de l'impérialisme occidental.

\section{La réussite du dirigisme asiatique}

Plus encore, les seuls pays qui sont parvenus à gagner des parts de marché considérables face aux entreprises occidentales sont des États qui ne sont ni démocratiques ni libéraux, dans le sens où nous l'entendons en Occident. Il s'agit bien sûr des pays asiatiques qui pratiquent un dirigisme très éloigné de la démocratie libérale. Comment expliquer la réussite de l'Asie? Les pays asiatiques entrent dans la mondialisation de manière totalement décomplexée et, surtout, ils ont un fonctionnement très différent de celui de nos sociétés occidentales fondées sur la démocratie et le libéralisme.

Prenons le cas de Singapour dont les caractéristiques du modèle de développement sont similaires à toutes celles des économies d'Asie. Ce pays est-il une démocratie au sens occidental du terme et une sorte d'îlot idéal du capitalisme, tel qu'on semble l'admettre aujourd'hui? En 1973, au moment où les accords de Paris mettent fin à la guerre du Viêtnam, Singapour est dirigé par Lee Kwan Yew, le chef du parti communiste, qui deviendra le principal artisan de son développement économique. Ceci peut sembler paradoxal: un communiste fondateur de la ville la plus capitaliste d'Asie! Ce n'est étonnant que si nous considérons le communisme d'un point de vue occidental. D'un point de vue chinois, il y a vingt-sept siècles que le confucianisme, c'est-à-dire

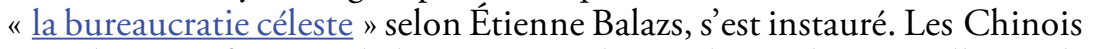
sont donc confrontés à la bureaucratie depuis des siècles. Par ailleurs, ils sont nourris de traditions villageoises collectivistes, issues de l'antiquité chinoise: propriété collective de la terre et organisation collective du travail. Au moment de l'arrivée du bouddhisme en Chine, un nouvel élan de collectivisme se manifestera à travers le développement des monastères qui s'occupent d'agriculture, d'artisanat et de reproduction des connaissances, de la même manière que les monastères chrétiens. Le collectivisme et la bureaucratie existaient donc un peu partout en Asie et il n'est pas étonnant que le communisme y ait trouvé un terreau d'accueil.

Que se passe-t-il sur le plan politique à Singapour? La principale doctrine asiatique, d'origine chinoise, est le confucianisme qui contient une philosophie du "gouvernement par la vertu». Elle consiste, pour l'Empereur, à écouter les conseils des sages, nous dirions aujourd'hui des experts, avant de prendre des décisions pour le bien du peuple, puis à les appliquer de manière autoritaire. Par exemple, parmi les actions qu'il fallait mettre en œuvre pour optimiser le développement de Singapour, une des solutions retenues était de faire de ses habitants les gens les mieux éduqués d'Asie. Sur les conseils d'experts, il a donc été mis en place un programme d'étude et les habitants ont été sommés de scolariser leurs enfants. Après cette convocation, les comportements des parents des enfants non scolarisés relèvent pratiquement de la haute trahison. Autre exemple, le droit du travail a été pratiquement supprimé pour attirer les investisseurs. En cas de plainte d'un salarié, la seule possibilité pour qu'elle soit examinée 
par la justice est qu'elle relève d'un problème d'intérêt collectif pour toute la communauté. Les droits individuels sont subordonnés à l'intérêt collectif. Dans tous les cas, l'application des directives politiques est d'autant plus autoritaire que les mesures sont prises pour le bien de la communauté.

Même autoritarisme sur le plan économique: comme la plupart des pays asiatiques, l'état de Singapour pilote des stratégies de spécialisation compétitive qui réunissent toutes les entreprises locales sur des secteurs (textile, électroménager, électronique grand public, etc.) qui sont développés grâce à des stratégies économiques nationales, directement projetées à l'échelle mondiale. Ces stratégies sont mises en place sous l'égide de l'État: il étudie l'évolution du marché mondial pour déterminer ses cibles, il investit de manière significative dans la recherche et le développement pour mettre à niveau ses entreprises locales, il prend des mesures réglementaires d'incitations fiscales, etc. C'est le contraire des règles libérales des économies occidentales, où les entreprises sont autonomes dans leurs décisions, où chaque entreprise monte seule en puissance sur son marché national, avant de se positionner de manière autonome à l'international.

En bref, que ce soit sur le plan politique ou économique, les méthodes du dirigisme asiatique ne ressemblent en rien aux pratiques occidentales et n'ont aucun rapport avec la démocratie et le capitalisme.

\section{Universalisme de l'intégrisme islamique?}

Pour couronner le tout, il nous faut parler d'une autre idéologie qui prétend à l'universalisme, celle de l'intégrisme islamique. La chute du mur de Berlin, puis celle de l'Union soviétique, ont soulevé l'espoir d'une paix mondiale durable: c'est la thèse de Fukuyama sur « la fin de l'Histoire ". Il ne faudra malheureusement que très peu de temps pour que l'urss cède à l'Islam le rôle d'ennemi de l'Occident. La rivalité Est/Ouest avait occulté la montée en puissance d'un ensemble de conflits dans lesquels le facteur commun était le rôle des pays musulmans: en 1979, la révolution théocratique de Khomeiny, puis l'invasion de l'Afghanistan par l'Unss, la guerre entre l'Irak et l'Iran, l'invasion du Koweït par les Irakiens, la montée du fis en Algérie, la deuxième Intifada du conflit israélo-palestinien, etc.

C'est alors qu'en 1995, Samuel Huntington, nous annonce un « choc des civilisations". Selon lui, les guerres du Xxi ${ }^{\mathrm{e}}$ siècle seront des guerres de civilisations. Huntington nous décrit une "connexion islamo-confucéenne ", c'est-à-dire une alliance de pays musulmans et asiatiques en train de s'armer pour former un front antioccidental. De plus, il désigne nommément l'Islam comme la principale force de déstabilisation mondiale de l'après-guerre froide. Selon lui, "le sang coule sur toutes les frontières de l'Islam ${ }^{3}$ ". Après l'optimisme économique de Fukuyama, voici le pessimisme militaire d'Huntington.

Le problème de cette thèse est tout simplement qu'elle est fausse. Il y a bien eu des guerres entre des peuples de civilisations différentes mais la violence est toujours plus grande dans un conflit à l'intérieur d'une même civilisation. Les guerres les plus violentes sont des guerres de proximité. C'est la proximité qui maintient à la fois les tensions en suspens et les prota- 
gonistes en présence. Ne prenons que l'exemple des deux guerres mondiales $\mathrm{du} \mathrm{xx}^{e}$ siècle qui ont été avant tout des guerres civiles européennes. $\mathrm{Ou}$, pour reprendre les exemples précédents, plus récents encore, les luttes fratricides entre Serbes, Croates et Bosniaques dans l'ex-Yougoslavie, celles entre Hutus et Tutsis au Rwanda, ou encore entre chiites, sunnites et Kurdes en Irak. Même dans les cas d'affrontements entre peuples de civilisations différentes, les conflits ont rarement des causes ou des objectifs culturels. La colonisation occidentale, par exemple, couramment justifiée par un «idéal civilisateur ", avait des enjeux économiques et géopolitiques beaucoup plus importants que ses justifications civilisatrices. Plus récemment, les conflits de "purification ethnique" auxquels nous avons assisté au Rwanda et dans l'ex-Yougoslavie, mettaient bien en jeu des facteurs culturels et même des discours racistes avoués, mais les objectifs concrets des affrontements étaient bien la répartition territoriale des différentes communautés ainsi que ses conséquences sur le plan du pouvoir politique et du développement économique.

En revanche, et même si la thèse d'Huntington est fausse, la menace, non de l'Islam, mais de l'intégrisme islamique et du terrorisme est réelle, bien que le danger ne soit pas nécessairement là où nous l'attendons. La plupart des islamologues considèrent que l'intégrisme islamique est un échec sur le plan politique et économique, c'est-à-dire dans sa capacité à construire un modèle alternatif de développement fondé sur des valeurs islamiques, à l'instar du dirigisme asiatique. Le terrorisme reste une menace à ne pas prendre à la légère mais, curieusement, la principale réussite actuelle de l'intégrisme islamique est d'ordre idéologique: c'est celui d'avoir imposé son universalisme, y compris aux démocrates qui le combattent.

En effet, à travers les affaires du voile islamique, de la fatwa contre Salman Rushdie, des caricatures de Mahomet, etc., les groupes intégristes ont réussi à imposer la prise en compte de leurs critères idéologiques aux démocraties occidentales. Prenons un seul exemple: celui des caricatures de Mahomet. Le droit à la caricature est reconnu partout en Europe mais, dès les premières protestations des groupes intégristes, nous voyons de belles âmes s'indigner: " Il y a des limites, on ne peut rire de tout, etc. ", et nous voyons toute la presse européenne s'incliner. En France, seul Charlie Hebdo sauve l'honneur.

Les intégristes ont remporté une victoire idéologique, ils ont imposé leurs critères idéologiques et religieux à pratiquement toute la presse européenne. Pour éviter cette défaite, il aurait fallu que toute la presse publie, dès le lendemain, des caricatures de Mahomet, du Christ, de Bouddha, etc. $\mathrm{Si}$ nous n'avons pas ce courage à l'avenir, celui d'affirmer les valeurs et les lois républicaines, notamment celui de la séparation de l'église et de l'état, alors nous ouvrons la porte à l'intégrisme, à l'universalisme du califat.

\section{Conclusion}

Récapitulons. D'abord, au moment où les Occidentaux pensent que la démocratie libérale est devenue universelle, cet universalisme autoproclamé est contesté par les trois quarts des pays membres de l'onu, pour lesquels les valeurs occidentales participent d'un néocolonialisme principalement destiné à préserver les intérêts occidentaux. Ensuite, les performances éco- 
nomiques des pays asiatiques des 30 dernières années ont été obtenues sur la base d'un dirigisme qui a très peu à voir avec le capitalisme et la démocratie tels qu'on les conçoit en Occident. Enfin, l'intégrisme islamique, malgré sa défaite politique et économique, malgré ses attentats terroristes, remporte des victoires idéologiques sur un Occident déboussolé qui ne sait plus défendre ses valeurs quand il importe vraiment de le faire face à la terreur. Dans ces conditions d'oppositions idéologiques, comment assurer la communication et le bon déroulement des négociations internationales dans une période ou l'interdépendance est la caractéristique majeure de la mondialisation? Mais ce n'est pas tout, car les querelles idéologiques ou identitaires sont une chose et la crise systémique à laquelle nous allons être confrontés à l'échelle mondiale en est une autre. Entre la guerre économique mondiale pour le contrôle des ressources naturelles qui bat son plein, la crise du pétrole, celle à venir de l'eau potable, le problème du changement climatique, le trou de la couche d'ozone, la multiplication des catastrophes naturelles, etc., notre modèle mondial de développement est confronté à une crise d'une ampleur et d'une rapidité de propagation inédite. Comble d'infortune, au moment où ce modèle occidental semble condamné, 1,4 milliard de Chinois, 1,2 milliard d'Indiens, 1,1 milliard de musulmans, 650 millions d'Africains, 450 millions de Latino-Américains et 250 millions de Slaves, revendiquent légitiment de voir leur niveau de vie s'élever sur les bases de ce modèle.

Compte tenu des défis mondiaux à relever dans les prochaines décennies, il est fondamental que nous puissions communiquer sans préjugés dans les négociations internationales. Depuis presque trente années, j'enseigne les civilisations dans les entreprises pour améliorer la communication, la négociation et le management dans un contexte mondial multipolaire et multiculturel. Mais cela ne suffit plus, car il ne s'agit plus aujourd'hui de négociation d'entreprises, mais de négociations globales, internationales et multilatérales. C'est pourquoi il nous faut rapidement apprendre à négocier en tenant compte des différentes visions du monde en présence et non en postulant a priori qu'une vision du monde universelle doit servir d'étalon international. D'autant plus que plusieurs visions « universelles » et autoproclamées sont en présence. Les différences culturelles sont un fait dont il nous faut aujourd'hui prendre la mesure. Il existe d'ailleurs des différences significatives en matière de démocratie et de capitalisme au sein même des pays occidentaux. Les démocraties bipolaires anglo-saxonnes se distinguent aussi bien des régimes sociauxdémocrates alémaniques et scandinaves, que des démocraties pluralistes du sud de l'Europe. De même, sur le plan économique, le capitalisme néolibéral à dominante financière des Anglo-Saxons se distingue assez nettement du libéralisme tempéré de la plupart des pays européens. Il faudra donc nous habituer à considérer que le $\mathrm{XxI}^{\mathrm{e}}$ siècle verra apparaître différentes formes de systèmes économiques et politiques sans nous en offusquer a priori. Ce qui ne veut pas dire que les Occidentaux doivent reculer sur leurs propres valeurs, mais qu'ils devront démontrer l'excellence de la démocratie libérale par l'exemple et non par l'idéologie. C'est ainsi que le retour en puissance des civilisations ne constituera pas une régression vers le passé mais, peut-être, la voie d'accès la plus légitime à 
des formes de démocratie et d'économie de marché adaptées aux valeurs des différentes aires civilisatrices.

\section{Notes}

* Docteur en philosophie, professeur de civilisations comparées, conseil en stratégie.

Vous pouvez également lire cette intervention ainsi que d'autres textes de Bernard Nadoulek sur le site personnel de l'auteur. $\pi$

1. Huntington, Samuel, Le Choc des civilisations, Paris, Odile Jacob, 1997; 2000.

2. Funuyama, Francis, "The end of History ", The National Interest, 1989;

repris dans Commentaire, $n^{\circ} 47,1989$.

3. Huntington, Samuel, Le Choc des civiliations, op. cit. \ 
Lundi 30 janvier 2006

\section{La mondialisation économique : chance ou fléau pour les pays pauvres?}

\section{Ouverture}

Brice Couturier

Commerce, ouverture, pauvreté

Lionel Fontagné

Commerce et lutte contre la pauvreté :

une équation à plusieurs inconnues (non disponible)

Céline Trublin

Bâtir un secteur financier ouvert à tous (non disponible)

Maria Nowak

Le paradoxe de la mondialisation en Afrique (à venir)

Stephen Smith

Débat (non disponible)

Modérateur : Brice Couturier 


\section{Ouverture}

\section{Brice Couturier*}

Le sujet que nous allons aborder s'intitule «La mondialisation économique: chance ou fléau pour les pays pauvres? " La rencontre précédente, "Qu'est-ce que la mondialisation?", a permis de définir un certain nombre de concepts de base qui vont nous permettre d'aller un peu plus loin dans notre réflexion. Aujourd'hui, la question est: l'intensification des échanges profite-t-elle aux pays pauvres? Cette interrogation rejoint deux autres questions qui sont habituellement posées dans les médias. Les pays du Sud, qui ont choisi l'ouverture des échanges commerciaux, en ont-ils bénéficié? Les inégalités entre pays riches et pays pauvres ont-elles augmenté ou décru en raison de cette mondialisation?

C'est un sujet d'une grande actualité puisque nous sommes toujours dans le cycle de Doha. La réunion de l'omc ${ }^{1}$, qui s'est tenue à HongKong en décembre 2005, nous a permis de comprendre à quel point les positions à l'intérieur même des pays émergents étaient divergentes. Un certain nombre d'entre eux demandent en effet la fin du protectionnisme, en particulier le protectionnisme agricole du Nord. D'autres, au contraire, craignant la ruine de leur propre paysannerie, ont exigé le maintien des subventions et des barrières tarifaires. L'affaire est donc beaucoup plus ambiguë que ne laisse croire un certain nombre de présentations rapides et idéologiquement orientées.

Je vais maintenant vous présenter les intervenants en respectant l'ordre dans lequel ils vont prendre la parole. Ils vont ainsi nous donner leur sentiment sur les effets de la mondialisation sur les pays pauvres à partir d'une grille d'analyse qui leur est propre.

Lionel Fontagné est professeur d'économie à l'université Paris ISorbonne et directeur du CEPII ${ }^{2}$. Le CEPII est l'un des grands centres d'analyses et de recherches sur le commerce international français.

Céline Trublin est directrice des campagnes Agir ici et membre observateur de la confédération oxfaM International ${ }^{3}$.

Maria Nowak est présidente de l'ADIE ${ }^{4}$ et préside également le Réseau européen de microfinance. Elle a notamment publié différents ouvrages à travers lesquels elle a exprimé ses positions sur la question du microcrédit et la façon dont le microcrédit peut aider le développement des pays du Sud. Elle a par ailleurs été conseillère spéciale auprès de Laurent Fabius lorsqu'il était ministre des Finances entre 2000 et 2002.

Stephen Smith est, quant à lui, écrivain et journaliste, spécialiste de l'Afrique. Il a obtenu le prix France Télévisions du meilleur essai pour son livre qui a connu un vif succès Négrologie, pourquoi l'Afrique meurt ${ }^{5}$. Son témoignage nous intéresse vivement puisque, lorsqu'il est question des pays pauvres, hélas, l'Afrique est encore aujourd'hui au centre de nos préoccupations.

Je vais commencer par donner la parole à Lionel Fontagné mais je voudrais, au préalable, faire une observation en introduction au débat. J'ai apporté ici un numéro du très vénérable et très institutionnel TLS, Times Literary Supplement, qui avait consacré le 18 mars 2005, un grand article sur différents ouvrages consacrés à la globalisation et donc à la mondialisation. Il était d'ailleurs question de deux livres français: celui de Jean-François Bayart, Le Gouvernement du monde ${ }^{6}$, 
une critique politique de la globalisation, et celui de Daniel Cohen, La Mondialisation et ses ennemis ${ }^{7}$.

De manière un peu provocatrice et une nouvelle fois dans la perspective d'introduire le débat, je vais essayer de traduire quelques-uns des premiers paragraphes de cet article:

"Le débat sur la mondialisation, tel qu'il a lieu dans de nombreux pays, rappelle celui qui se déroule autour du darwinisme aux ÉtatsUnis. Dans les deux cas, il existe un fossé abyssal entre le savoir de base et l'état des discussions publiques. [...] Les médias décrivent l'orthodoxie libre-échangiste comme si c'était une idée soumise à une véhémente discussion. Mais le fondement de cette idée, la loi ricardienne de l'avantage comparatif - qui montre pourquoi il est sensé, même pour les pays qui ne disposent pas d'avantage naturel, de se spécialiser et d'échanger - n’a, en réalité, jamais été sérieusement contesté. Ce qu’on ne soupçonnerait pas, à se contenter de la lecture des journaux, qui tendent à offrir une représentation faussée des débats universitaires sur le commerce.»

On pourrait s'amuser à opposer les ouvrages des économistes - qui font, le plus souvent, un éloge des bénéfices supposés de la mondialisation - à ceux des sociologues, qui la rendent, au contraire, responsable d'un certain nombre de dégâts humains, comme Zygmunt Bauman ${ }^{8}$. De nombreux politologues l'accusent en outre de provoquer une dépossession démocratique, en plaçant les vrais décideurs hors d'atteinte des États. La question de la mondialisation doit en conséquence bénéficier d'un éclairage multiple, s'appuyant sur toutes les disciplines des sciences humaines, afin de nous livrer l'essentiel de ses enjeux.

\section{Notes}

* Producteur à France Culture, maître de conférences associé en sciences politiques à l'université de Marne-la-Vallée. $\pi$

1. OMC: Organisation mondiale pour le commerce. $\boldsymbol{A}$

2. CEPII: Centre d'études prospectives et d'informations internationales. $\boldsymbol{\lambda}$

3. oxfam: Oxford Commitee for Famine Relief. $\boldsymbol{\lambda}$

4. ADIE: Association pour le droit à l'initiative économique. $\pi$

5. Sмiтн, Stephen, Négrologie, pourquoi l'Afrique meurt, Paris, Calmann-Lévy, 2004. $\rtimes$

6. BAYART, Jean-François, Le Gouvernement du monde. Une critique politique de la mondialisation, Paris, Fayard, 2004. $\pi$

7. Cohen, Daniel, La Mondialisation et ses ennemis, Paris, Grasset, 2004. $\lambda$

8. Bauman, Zygmunt, Le Coût humain de la mondialisation, Paris, Hachette Littérature, 1999. $\pi$ 


\section{Commerce, ouverture, pauvreté}

\section{Lionel Fontagné *}

Je vous remercie pour cette introduction, mais je ne voudrais pas laisser croire au public présent que les économistes dressent tous un panorama aussi idyllique de la situation. En réalité, la profession dans son ensemble a, aujourd'hui, une vision assez pondérée de la mondialisation. Aucun économiste n'affirmera que la mondialisation n'entraîne aucun coût pour toutes les catégories de pays concernés.

Nous reviendrons peut-être, à travers cette discussion, sur les positions de Paul Samuelson relatives à la question des délocalisations. Je voudrais, à cet effet, rappeler que Paul Samuelson est l'économiste qui a developpé la forme la plus épurée de la théorie classique du commerce international, des gains, etc. Il a, durant 30 ou 40 ans, formalisé et construit l'édifice qui est aujourd'hui critiqué. La critique qu'il apporte lui-même à cette théorie est liée aux délocalisations dont nous allons reparler par la suite.

Pour résumer ce point introductif, les économistes sont aujourd'hui beaucoup plus lucides et pondérés sur les bénéfices de la mondialisation que l'opinion publique ne le dit. Ils ne sont cependant pas contre la mondialisation. Elle est un fait qui s'impose aux politiques économiques, elle n'est ni une chance ni un fléau, elle doit être comprise et accompagnée.

Je voudrais maintenant vous présenter brièvement le CEPII ${ }^{1}$, vous pourrez trouver sur son site toutes les informations que je n'aurai pas le temps de vous donner. Le CePII est un centre qui fait de la macroéconomie, de la finance et du commerce international. Ce centre s'est récemment illustré en mettant sur la place publique la question des préférences commerciales. Je reviendrai sur les préférences commerciales dans la suite de mon intervention. Le CePII a remis en cause l'idée selon laquelle les pays les plus pauvres sont ceux qui vont gagner le plus au cycle. C'est une idée qui était très répandue il y a quatre ou cinq ans et que plus personne n'ose défendre aujourd'hui. Nous avons contribué très modestement à faire reculer cette idée, mais nous ne sommes pas pour autant opposés à la mondialisation. Comme je l'ai expliqué précédemment, nous considérons que la mondialisation est un fait et qu'il faut essayer de la comprendre, et de l'accompagner.

L'autre élément important qu'il faut avoir à l'esprit, c'est la dimension historique de la mondialisation. Afin d'analyser la situation actuelle, il est important d'évoquer les précédents épisodes de la mondialisation dans l'Histoire, notamment le grand épisode de la mondialisation du XIX ${ }^{e}$ siècle dont les modalités ont été différentes, mais dont les conséquences ont elles aussi été considérables. Il est également important de savoir que la mondialisation découle de deux forces. La première est une force à caractère technique qu'il n'est pas possible d'arrêter. Elle s'exprime par la réduction des coûts de communication, avec entre autres Internet. La seconde force est quant à elle institutionnelle. Le multilatéralisme et la mondialisation sont des choix politiques faits par des gouvernements élus. Il est important de comprendre ceci car ces choix sont parfaitement réversibles.

Les différents épisodes de la mondialisation auxquels j'ai fait référence montrent qu'il y a eu, dans l'histoire économique mondiale, des allers et retours du libre-échange vers le protectionnisme. Le mouvement du protectionnisme vers le libre-échange ne s'est donc pas déroulé de manière univoque. Voici donc un tableau général à caractère introductif. 
Vous savez certainement que le commerce mondial progresse très vite; en réalité, il progresse presque trois fois plus vite que le revenu mondial. À chaque fois qu'un dollar ou un euro est créé dans le monde, il y a trois dollars ou trois euros de commerce en plus. Ce phénomène s'est accéléré récemment. Les années 2000 marquent un tournant. La contribution majeure à cette progression des échanges est le fait d'une catégorie de pays, les pays émergents. Les pays émergents tirent aujourd'hui les exportations mondiales, ce sont eux les vendeurs, mais ils tirent également les importations mondiales, ce sont donc aussi les acheteurs. De plus, les ventes à l'étranger des filiales des firmes multinationales représentent deux fois le volume des exportations mondiales. Lorsque nous parlons de la mondialisation, nous pensons aux exportations, mais les ventes sur place des filiales de ces firmes représentent deux fois ce montant. Environ 50 millions de personnes sont employées dans plus de 50000 firmes multinationales qui détiennent à elles seules 100000 filiales dans le monde. Cette présence à l'étranger, dont on comprend l'importance, n'est pourtant pas dans les chiffres du commerce international. Le commerce international n'est, en conséquence, qu'un aspect de la mondialisation.

Comme Brice Couturier l'a expliqué en introduction, la société civile pense que les bénéfices de la mondialisation ont été exagérés par les économistes alors que les coûts ont été sous-estimés. L'idée que la mondialisation a véhiculé la misère dans de nombreux pays en voie de développement - notamment le travail des enfants, les bas salaires ou encore l'exploitation -, est très présente dans la société civile. L'idée que les économistes n'ont pas suffisamment ces questions en tête est également très présente.

En réalité, le diagnostic des économistes n’est pas celui-là. Trois éléments constituent le diagnostic devant être fait de la mondialisation: l'ouverture économique est préférable, même si elle n'a pas que des avantages; tous les pays qui se sont ouverts n'ont pas participé au grand banquet de la croissance et de la mondialisation - parmi les pays du Sud, certains en ont beaucoup profité alors que d'autres n'en ont pas profité du tout -; enfin, il est difficile de dissocier dans la capacité différenciée des pays du Sud à bénéficier de la mondialisation, ce qui relève de la responsabilité du Sud - de mauvaises institutions, une mauvaise gouvernance - et ce qui relève de la responsabilité du Nord - le protectionnisme. Je pointe du doigt le protectionnisme du Nord au niveau de l'agriculture et des industries de main-d'œuvre, comme le textile et l'habillement. Repensez à ce qui s'est passé avec le textile chinois récemment.

Le sujet nous intéressant aujourd'hui me semble avoir trois dimensions. Je vais de ce fait répondre en trois points à la question qui m’a été posée. Louverture du commerce international entraîne-t-elle systématiquement la croissance? Et si ce n'est pas systématique, à quelles conditions l'ouverture n'entraîne-t-elle pas la croissance? A supposer que la réponse soit positive, la croissance qui est consécutive à une ouverture va-t-elle profiter d'abord aux plus défavorisés? Les pays en voie de développement tirentils systématiquement bénéfice de la mondialisation? Brice Couturier a déjà répondu à cette question dans l'introduction, la réponse est non. Nous allons examiner ensemble pour quelles raisons. 


\section{L'ouverture entraîne-t-elle la croissance?}

Les faits ont tranché. Nous avons tous entendu parler du " miracle asiatique ". Cet archétype de l'émergence semble montrer que les pays en voie de développement qui ont fait le choix de l'insertion dans le commerce international s'en sortent plutôt mieux. Les économistes ont tous à l'esprit, tout en sachant que le monde est moins simple, que certains de ces pays asiatiques, ayant bénéficié du commerce international pendant une première période, se sont tout de même très largement protégés pour organiser leurs industries. Nous ne sommes pas assez naïfs pour ignorer ce phénomène; cependant l'ouverture leur a plutôt profité. Le deuxième point qui semble plaider dans ce sens est le fait que les pays en voie de développement, excepté quand le FMI est venu le leur demander, ont choisi d'ouvrir leurs frontières de façon presque autonome. En conséquence, cette option n'est probablement pas mauvaise. La troisième idée qui circule beaucoup chez les économistes est l'idée que les épisodes d'ouverture dans l'Histoire se sont accompagnés d'épisodes de croissance; et réciproquement, les épisodes de fermeture ont plutôt été des épisodes de moindre croissance. En tout cas au $\mathrm{xx}^{\mathrm{e}}$ siècle. Par conséquent, l'idée partagée par les économistes - idée reprise par Brice Couturier en introduction - est que l'ouverture est préférable. L'échec des politiques autocentrées le confirmerait si besoin.

Ce qui précède ne signifie pas l'existence d'un seul modèle de développement. Je viens d'expliquer que certains pays asiatiques avaient pris des "libertés" avec le libre-échange, au moins dans un premier temps, et surtout que l'ouverture n'est pas systématiquement amorcée dans les pays en développement - ce que les historiens appellent le take off ou le décollage de l'économie. Certains pays s'étant ouverts et spécialisés pour des produits dont le prix baissait au niveau mondial de façon tendancielle, ou pour des produits qui débouchaient généralement sur une mauvaise gouvernance, n'ont pas du tout bénéficié de l'ouverture. Lorsqu'un pays exporte des diamants, il rencontre généralement des difficultés car la gouvernance adéquate est très difficile à mettre en place. Notre seule certitude réside donc dans l'idée que l'ouverture économique est globalement préférable, que les politiques de fermeture seraient probablement pires dans tous les cas. Beaucoup de travaux statistiques ont été faits pour essayer de comprendre les mécanismes par lesquels l'ouverture pouvait nourrir la croissance. Ces travaux indiquent que l'ouverture fonctionne lorsqu'elle est associée à la scolarisation, à un investissement privé sur place et à une épargne intérieure. S'il manque l'un de ces éléments, l'ouverture ne fonctionne pas.

\section{La croissance liée à l'ouverture}

profite-t-elle aux plus défavorisés?

Afin de comprendre ce deuxième point, je vais procéder à un rappel des mécanismes qui se mettent en place lorsque l'on ouvre une économie au libre-échange.

Le libre-échange permet en premier lieu d'accéder à des prix plus favorables, alors que le protectionnisme implique de payer plus cher les biens auxquels la protection s'applique. À travers l'ouverture 
économique, les ressources s'orientent vers les emplois les plus efficaces. Je vous l'énonce de façon provocatrice pour lancer la discussion qui va suivre. L'ouverture induit aussi un renforcement de la concurrence. Les producteurs et les consommateurs sont satisfaits parce qu'ils ont accès à une plus grande variété de produits. L'ouverture de l'économie permet de pouvoir acheter non seulement les variétés produites sur place mais également de pouvoir acheter des variétés produites ailleurs, des produits, des services et des technologies nouvelles. L'ouverture permet par conséquent d'accéder au grand panier de la technologie disponible sur le marché mondial. Un pays relativement pauvre, qui ne dispose pas de la technologie correspondante, peut donc se procurer des ordinateurs sur le marché mondial.

Je viens d'énoncer les aspects favorables du libre-échange; néanmoins le prix à payer en retour peut être élevé. Il réside dans les ajustements de l'économie que ces pays vont devoir mettre en place. Ajuster l'économie signifie qu'il va y avoir des coûts d'ajustement. Une redistribution des revenus va découler de l'ouverture commerciale. L'ouverture fait évidemment des gagnants, mais elle fait aussi des perdants. Les économistes le disent régulièrement, même s'ils ne sont pas toujours entendus. L'enrichissement moyen d'une économie ne profite pas nécessairement aux plus pauvres dans cette économie. Tout dépend des circonstances de la mise en place de cette ouverture. L'ouverture peut générer des inégalités, y compris dans les pays qui s'en sortent très bien.

C'est le cas des pays émergents qui croissent rapidement, dont le revenu par tête augmente très vite en moyenne, mais dans lesquels les inégalités sont de plus en plus criantes. La difficulté que les économies des pays pauvres rencontrent est l'absence d'institutions permettant d'accompagner l'ouverture, de redistribuer le revenu et de s'occuper des perdants. Ces pays ne disposent pas non plus des canaux de financement nécessaires, des marchés financiers permettant de déplacer les ressources d'un emploi vers l'autre. Lorsque les conditions nécessaires à l'ouverture ne sont pas réunies, lorsqu'il n’y a pas de marché pour les capitaux, lorsqu'il n'y a pas de filet social, lorsqu' il n'y a pas de redistribution des revenus, l'ouverture peut avoir des effets très inégalitaires. Il n'existe pas de mécanismes naturels qui font que les gagnants indemnisent les perdants. Lorsque les politiques correctrices sont absentes, l'ouverture peut avoir des conséquences sociales non désirées. Je viens ici de décrire les mécanismes liés à l'ouverture commerciale à l'intérieur des pays pauvres.

Nous allons maintenant nous intéresser aux effets de la mondialisation sur les inégalités entre les pays. Avec la globalisation, les inégalités entre pays au niveau mondial ont plutôt eu tendance à s'accroître, ce qui ne signifie pas que certains pays sont devenus plus pauvres; cela signifie que l'ascenseur est monté pour tous les pays, mais pas à la même vitesse. Par conséquent, les écarts de niveau de vie entre les pays ont eu tendance à s'élargir. Certains pays très peuplés ont rattrapé très vite les autres pays. Si l'on pondère le revenu de ces pays par leur population, l'affirmation précédente n'est plus exacte: l'ascenseur des pays les plus pauvres est donc monté plus vite que celui des pays les plus riches. Ce phénomène est uniquement lié à l'émergence de la Chine. 


\section{Les pays en voie développement}

constituent-ils un groupe homogène?

Une grande difficulté des pays en voie de développement est d'être spécialisés soit dans l'agriculture, soit dans les industries de main-d'œuvre, secteurs dans lesquels les pays du Nord sont les plus protectionnistes. En raison de leur spécialisation dans l'agriculture, dans le vêtement et dans les jouets, les pays du Sud font face à plus de protection de la part du Nord. Néanmoins ces pays ne sont pas tous dans une situation identique vis-à-vis de ce problème; certains font face à un protectionnisme élevé, alors que pour d'autres il est moindre. La moyenne des droits de douane dans le monde, tous pays et tous produits confondus, est de $5 \%$. La moyenne de la protection dans le monde pour les produits agricoles est de $20 \%$, soit quatre fois plus. Il s'agit d'une moyenne; certains produits sont taxés à $100 \%$ de protection, d'autres à $150 \%$, $200 \%$ ou encore $300 \%$. La moyenne de la protection dans le textile et l'habillement est de $10 \%$.

Au-delà de ces moyennes, le principe général dans le commerce mondial est que les pays en voie de développement ont obtenu un traitement plus favorable, consistant en des droits de douane moins élevés que ceux appliqués à d'autres pays. Cela dit, tous les pays n'ont pas la même attitude vis-à-vis des pays en voie de développement. L'Union européenne se singularise - n'oublions pas que l'Union européenne est considérée comme un pays du point de vue du commerce international puisqu'il existe un tarif extérieur commun: elle propose de façon unilatérale des conditions d'accès plus favorables aux pays en voie de développement. Ces conditions ne s'appliquent toutefois qu'aux pays les plus pauvres et ne concernent pas tous les pays en voie de développement. Cette politique peut paraître satisfaisante d'un point de vue moral, mais elle pose néanmoins des problèmes, notamment pour les pays à revenu intermédiaire. Or, dans des pays à revenu intermédiaire, comme le Brésil, il existe tout de même beaucoup de pauvres. C'est le reproche que la Banque mondiale fait à l'Union européenne.

L'Union européenne applique donc en moyenne un droit de douane vis-à-vis des pays les plus pauvres - appelés également "pays les moins avancés" - à peu près égal à zéro. Certains produits tels que le riz, la banane et le sucre forment une exception appelée à disparaître. Un calendrier a été mis en place et, dans un horizon proche, ces produits seront libéralisés. Les pays pauvres paieront $0 \%$ de droit de douane, feront face à zéro quota et pourront exporter tous les produits qu'ils désirent, à partir du moment où ils sont fabriqués dans le pays, à l'exception des armes, d'où l'appellation "Tout sauf les armes ". Ce processus pose tout de même des difficultés, notamment la question des règles d'origine: si un pays fabrique des chemises sur place mais utilise du tissu acheté ailleurs, il est difficile de donner un statut local au produit et il ne pourra pas bénificier des avantages précédemment évoqués.

La situation est très différente aux États-Unis. Les programmes pour les pays les plus pauvres, appelés AGOA, sont moins efficaces que les programmes européens, même si l'on perçoit des effets dans le domaine du textile/ habillement, en raison cette fois de la générosité des règles d'origine. 
Certains pays disposent de préférences quand ils accèdent à des marchés. Ils ne paient rien alors que d'autres pays paient $10 \%, 20 \%, 30 \%$ et donc cette marge compétitive de $5 \%, 10 \%, 20 \%$ est un bénéfice pour les pays qui en profitent.

Les discussions actuelles à Hong-Kong portent sur la baisse des tarifs de façon multilatérale. La marge de préférence dont disposaient les pays qui bénéficiaient des politiques venant d'être évoquées va se réduire. La libéralisation du commerce va donc entraîner des situations où certains pays en voie de développement seront favorisés et d'autres défavorisés. Ce phénomène engendre une érosion des préférences et pose des difficultés.

\section{Certains pays vont-ils gagner plus que d'autres?}

Le montant des préférences initiales joue un rôle important sur ce que certains pays peuvent gagner. Plus ces pays bénéficient initialement de préférences, moins ils vont gagner dans l'avenir, puisque le multilatéralisme réduit ces préférences. Le deuxième mécanisme déterminant l'ampleur des gains attendus réside dans l'évolution des prix mondiaux des produits agricoles: ces prix vont augmenter. Libéraliser la politique agricole commune, la politique des États-Unis, du Japon, ou de la Corée-du-Sud, fait croître les prix mondiaux: l'offre de ces pays diminue et leur demande augmente. Les pays avantagés par ce système sont les pays exportateurs nets de produits agricoles, qui vendent plus de produits agricoles sur le marché mondial qu'ils n'en achètent, notamment le Brésil et l'Argentine. Les pays pour lesquels la libéralisation est défavorable sont dans une situation opposée: ils achètent plus de produits sur le marché mondial qu'ils n'en vendent, en particulier les pays de l'Afrique subsaharienne.

Nous sommes bien conscients de la difficulté que posent ces négociations. Les pays intermédiaires ont beaucoup à gagner dans ce cycle de libéralisation parce qu'ils faisaient face à une très forte protection. Ils ont beaucoup à gagner avec la baisse des droits de douane et aussi beaucoup par rapport aux prix mondiaux car ils sont producteurs nets de produits alimentaires. À l'inverse, certains pays perdent sur les deux tableaux: les pays d'Afrique subsaharienne ne sont pas, en moyenne, des exportateurs nets. De plus, ces pays vont devoir faire face à l'érosion de leurs préférences.

Les mécanismes venant d'être évoqués ne justifient toutefois pas le maintien du protectionnisme. Le protectionnisme favorise avant tout les systèmes de rente. En particulier, en présence de quotas d'importation, les détenteurs de licences d'importation sont ceux qui profitent le plus du protectionnisme, dans les pays en voie de développement comme ailleurs.

Pour conclure, la thèse classique de la " convergence économique " est remise en cause de façon définitive par la situation de nombreuses économies en développement, qui ont des difficultés persistantes. La notion de convergence est également remise en cause par le fait que de fortes inégalités mondiales persistent.

Nous avons vu que les gains directs d'ouverture sont en fait assez limités pour certaines catégories de pays en voie de développement; mais nous n'avons pas encore évoqué la dimension politique et sociologique 
de l'ouverture. Cette dimension est importante dans la mesure où ouvrir une économie équivaut, d'une certaine façon, à se lier les mains par rapport à la communauté internationale et à essayer en général d'améliorer sa gouvernance. Ce second aspect apporte beaucoup plus que l'ouverture elle-même.

Le commerce à lui seul ne va donc pas résoudre tous les problèmes des pays en voie de développement et il faut mettre en place d'autres solutions. Nous connaissons ces solutions. Ces solutions sont les aides. Ces aides, qui posent elles aussi de nombreuses questions, sont destinées aux pays en voie de développement qui ne vont pas participer, pour toutes les raisons que j'ai évoquées, au grand banquet de la mondialisation. Je vous remercie.

\section{Notes}

* Professeur d'économie à l'université de Paris I-Sorbonne. $\pi$

1. Voir le site Internet du CEPII. 7 


\title{
Lundi 20 février 2006
}

\section{Les pays riches dans une économie mondialisée}

\author{
Ouverture \\ Philipe Manière
}

Compétitivité mondiale, cohésion sociale

et modèle social européen sont-ils compatibles? (non disponible)

Joël Décaillon

\section{Mondialisation et territoires}

Philippe Martin

Pourquoi les Français se méfient

du capitalisme financier (non disponible)

David Thesmar

Vers une mondialisation socialement responsable (à venir) Thierry Verdier

Débat (non disponible)

Modérateur : Philippe Manière 


\section{Ouverture}

\section{Philippe Manière*}

Bonsoir à tous et merci à d'être présents pour assister à cette séance. Très rapidement, je vais commencer par me présenter: je suis directeur de l'Institut Montaigne qui est ce qu'on appelle en anglais un think tank. Il s'agit d'une organisation indépendante qui fait de la recherche et formule des propositions pour les décideurs publics dans trois domaines: le social, l'économique et la réforme de l'État. Je vais à présent vous donner quelques indications sur le déroulement du débat, en commençant par vous présenter nos invités. Je ne suis que l'animateur, mais nous avons la chance d'avoir quatre spécialistes qui ont préparé leurs interventions avec à chaque fois un point de vue différent.

S'exprimeront donc au cours de cette rencontre un syndicaliste, Joël Decaillon, secrétaire de la Confédération européenne des syndicats, ainsi que trois enseignants-chercheurs: David Thesmar, professeur associé d'économie et de finance à HEC et à Polytechnique, Thierry Verdier, directeur scientifique de Paris-Jourdan en sciences économiques et directeur d'études à l'École des hautes études en sciences sociales, et, enfin, Philippe Martin, qui est professeur d'économie à l'université de Paris I-Sorbonne. Chacun des intervenants donnera un éclairage différent sur le programme de la journée: "Les pays riches dans une économie mondialisée ". Le public aura alors l'occasion de relancer ces intervenants avec une ou deux questions qui auront pour but de préciser certains éléments. Enfin, je ferai vivre le débat entre eux avant de passer au jeu des questions-réponses. Ces dernières ont été très nombreuses dans les précédentes éditions. J'étais assez impressionné que, sur les trois heures de rencontre, un tiers du temps soit consacré à cet exercice mais voyant votre nombre, je me dis que cela ne sera pas de trop.

La mondialisation est un thème particulièrement intéressant parce que l'on oublie toujours, dans nos pays riches, qu'elle est assez populaire dans les pays en voie de développement, et en tout cas dans ceux qui en ont profité pour se développer. Certaines exceptions existent comme l'Argentine, qui n'est pas vraiment un pays en voie de développement mais qui a fait un grand pas en arrière, en grande partie à cause de la mondialisation, ou l'Indonésie, qui a commencé sa mondialisation au moment de la crise asiatique. Mais, pour les pays qui sont sortis du dénuement grâce à cette mondialisation, parmi lesquels Taiwan ou la Corée-du-Sud, l'internationalisation des échanges peut être considérée comme positive.

En revanche, elle est contestée dans les pays riches et, probablement, nulle part autant qu'en France. Depuis une dizaine d'années, cette contestation de la mondialisation est solidement établie et, à ce titre, le fait que le mouvement ATTAC, désormais international, soit né en France est assez révélateur. La position française est à l'image des pays développés, plus contestataires que les pays qui ne le sont pas, et notre pays est à la pointe de ce mouvement. Ceci est largement lié à l'idée que les gens se font de l'impact de la mondialisation sur les niveaux de vie et de protection sociale, généralement très supérieurs à ceux du reste du monde pendant des décennies. De mauvais arguments se dressent contre la mondialisation, comme il en existe contre tout phénomène, mais ils sont assez répétés pour que je les rappelle. A contrario, il y a également de bons 
arguments, au sens d'acceptables et de discutables. C'est sur ces points que nous allons nous concentrer.

Parmi ceux-ci, le plus intéressant repose sur le primat du financier. Ce qui revient à dire qu'aujourd'hui la finance dirige tout, qu'elle a pris le pas sur l'économie réelle. Faits incontestables, la mobilité des capitaux s'est considérablement accrue durant ces dernières décennies et l'identité des actionnaires a changé puisque les personnes physiques ont été remplacées par des organisations spécialisées, notamment les fonds de pension. Il faut également ajouter qưil en résulte une prospérité caractéristique, très concentrée dans les bénéficiaires et dans l'espace. Ceci donne l'impression d'une sphère où les personnes, très riches, qui travaillent dans la finance, sont déconnectées de la réalité. Le deuxième reproche est la désertification et l'appauvrissement de certains territoires. Avant d'en débattre, nous ne pouvons que constater que la mondialisation a vocation à accélérer ce qui s'appelle, en économie, la " destruction créatrice ". Ce qui revient à dire que de tout temps, des secteurs disparaissent du fait de l'évolution technique et de l'apparition de nouveaux concurrents. C'est une donnée éternelle, mais qui est probablement accélérée par la mondialisation. Et il faut bien reconnaitre que les dégâts sont importants dans certaines régions du monde, notamment dans notre pays. D'où la question des conséquences asymétriques de cette mondialisation sur les secteurs industriels. Le troisième argument réside dans la menace que ferait peser la mondialisation sur notre modèle social. Sans faire de simplisme, et même si ce n'est pas le débat de ce soir, je rappelle que nombre de pays encore sous-développés il y a 30 ou 40 ans bénéficient d'une évolution sociale favorable avec la création de syndicats et l'acquisition de droits. Mais ces derniers, conquis de haute lutte dans nos pays riches, vacillent désormais sous le poids d'une compétition accrue. Le quatrième point, lié au précédent, veut que la mondialisation change la donne entre gagnants et perdants, tant au niveau des territoires quà celui des individus et des industries. Ce qui impliquerait une adaptation de notre système de redistribution. Il s'agit là plus de conséquences de la mondialisation que d'un éventuel reproche à lui adresser. Ces quatre arguments ne sont pas idéologiques et peuvent être débattus librement et calmement.

Commençons donc par écouter Joël Decaillon sur le thème de la compatibilité de la compétitivité mondiale, de la cohésion sociale et du modèle social européen.

Notes

* Directeur de l'Institut Montaigne et chroniqueur à BFM. $\lambda$ 


\section{Mondialisation et territoires}

\section{Philippe Martin*}

Je vais donc aborder le thème du lien entre commerce international et inégalités régionales ou spatiales par le biais spécifique de la géographie. En fait, certains territoires peuvent se trouver affectés de manière différente, soit en bénéficiant de la mondialisation, soit en en pâtissant. Pour cela, il est intéressant de revenir sur des débats qui avaient eu lieu à la fin des années 1980 et au début des années 1990 et qui portaient sur l'intégration commerciale du grand marché en Europe. La crainte, à cette époque, était fondée sur la possibilité d'une grande divergence entre les "pays-cœur ", c'est-à-dire les pays riches, et les nations de la périphérie, à savoir l'Irlande, le Portugal, l'Espagne, la Grèce puis, plus tard, les pays de l'Europe de l'Est. On redoutait que ce grand marché, caractérisé par une spécialisation des pays et un phénomène d'agglomération des activités économiques sur certains pôles géographiques européens, n’aboutisse à une géographie similaire à celle des États-Unis. Ce qui posait de fait un problème d'équité entre ces pays.

\section{Mobilité}

Ce problème devient spécifique dès lors qu'il n'y a pas de mobilité entre les régions, ce qui est le cas en Europe. Car si les firmes et le capital vont s'agglomérer ou se délocaliser dans certaines régions en laissant sur place leurs travailleurs, il va se produire une baisse de la demande de travail alors que l'offre reste la même. Ce qui a pour conséquence de créer du chômage et des baisses de revenus. En revanche, si la mobilité est effective, si les travailleurs suivent, la demande et l'offre de travail diminueront et nous serons peut-être confrontés à un problème de régions vides. N'existera-t-il pas des inégalités de chômage entre les différentes régions? Alors pourquoi, en cas de phénomène d'intégration commerciale, l'activité se concentre-telle uniquement sur quelques régions avec, pour corollaire, l'augmentation des inégalités? S'il est facile pour les entreprises d'importer et d'exporter, elles vont exploiter les économies d'échelle, à savoir concentrer toute leur production sur quelques endroits seulement. L'intégration commerciale facilite leur tâche et elles n'ont plus besoin d'être géographiquement proches de leurs consommateurs finaux ou d'autres entreprises. En fait, c'est l'une des nouvelles dimensions de l'image de l'entreprise sDF évoquée précédemment. Cette crainte s'est-elle effectivement matérialisée en Europe?

\section{Convergence globale et divergence locale}

Tout cela ne s'est pas forcément déroulé de la manière prévue puisqu'il est apparu un phénomène de convergence globale et de divergence locale. Contrairement à ce que nous craignions, nous avons constaté un phénomène de convergence entre les pays relativement pauvres et ceux relativement riches, comme le démontrent les exemples de l'Irlande, de l'Espagne et maintenant des pays de l'Est. L'intégration commerciale n'est certainement pas le seul élément décisif pour expliquer cette convergence mais elle a incontestablement joué un rôle important. Ce à quoi nous nous attendions moins, c'est l'apparition d'une divergence à l'intérieur des pays. En effet, les inégalités ont augmenté entre les régions des grands pays européens. De ce point de vue, la production est devenue plus concentrée, non pas entre les pays, mais bel et bien entre les différentes régions. 
L'une des raisons pour expliquer ce phénomène de convergence globale et de divergence locale tient au fait que, si certains pays ont des coûts de production plus faibles, la production sera délocalisée, notamment vers les pays de l'Est, et c'est ce qui va organiser la convergence. Ce n'est pas le cas à l'intérieur même des pays puisqu'il existe très peu de différences de coûts de production. Ainsi, en France, les salaires sont assez similaires selon les régions. La concentration de la production s'est bien effectuée dans les régions à gros marchés dans le but d'être le plus proche possible des consommateurs finaux. Ce qui est assez facile à réaliser lorsque l'on est dans un grand marché intégré comme celui, par exemple, de 1992. Cela se vérifie en France mais également en Espagne. Des régions comme la Catalogne ou celles proches de Madrid, initialement les plus riches, sont celles qui ont eu le taux de croissance le plus élevé et elles ont convergé vers l'Europe. Ce sont les régions les plus riches qui ont profité au mieux de l'intégration européenne. Cette remarque vaut également pour la Pologne qui, même si son rythme est mouvementé, converge à son tour vers l'Europe. Là encore, ce sont les régions les plus riches, telles celles autour de Varsovie, qui ont le plus profité de cette intégration. Parallèlement, les inégalités en termes de taux de chômage ont augmenté entre les régions.

\section{Inégalités territoriales}

Cela s'explique par le fait qu'en Europe, contrairement à la géographie états-unienne, il existe peu de migrations entre les régions. Trois points importants sont à noter quant à ces phénomènes d'inégalités régionales et d'agglomération au profit des régions les plus riches et au détriment de celles qui étaient déjà initialement défavorisées. En premier lieu, il doit y avoir un arbitrage au niveau spatial, géographique entre équité et efficacité. Même si cette agglomération pose des problèmes en termes d'inégalités, elle possède également un certain nombre d'avantages. Il y a des intérêts économiques à être regroupés. Ainsi, par exemple, des économies peuvent être réalisées sur les coûts de transports puisque fournisseurs et clients sont proches. C'est incontestablement un gain d'efficacité. De même, beaucoup d'études démontrent que la proximité des entreprises qui évoluent dans un domaine permet de favoriser un échange des connaissances. C'est ce qu'on appelle les spinover technologiques. Le secteur aéronautique qui s'agglomère autour de Toulouse illustre parfaitement cet exemple.

Le deuxième point est le constat qu'on ne retrouve pas cette augmentation des inégalités dans la géographie des revenus disponibles, c'est-àdire ceux après impôts, transferts sociaux, etc. La production est devenue plus concentrée, par exemple en France, engendrant plus d'inégalités de ce point de vue, mais elle est devenue plus égalitaire au niveau des revenus disponibles. Il y a donc un phénomène de "ciseau ". De nombreux transferts entre les régions en France, comme les impôts sur le revenu ou les retraites, et l'État providence jouent un rôle important pour limiter et inverser le mouvement des inégalités entre les territoires français. Ce qui est vrai pour les régions ne l'est pas au niveau microspatial comme celui des quartiers où est constatée une augmentation des inégalités entre banlieues et villes. De même, la remarque précédente ne vaut pas pour 
tous les pays européens. En Grande-Bretagne, la production s'est concentrée de plus en plus dans quelques régions riches et cette augmentation des inégalités de production a été accompagnée d'une augmentation des inégalités de revenu disponible.

La manière dont les politiques réfléchissent aux inégalités territoriales a pour but de diminuer également les inégalités individuelles. Ce n'est pas toujours vrai lorsqu'il s'agit de politiques régionales ou d'aménagement du territoire. Elles ont été mises en place en Europe pour justement contrer ces phénomènes de concentration spatiale. Dans la pratique, cela se résume souvent à une subvention au capital en incitant les entreprises à se délocaliser dans les régions les plus défavorisées. Le problème est que ce capital est de plus en plus mobile du fait de l'intégration commerciale et financière, et que si le rendement s'égalise, il ne pourra plus être très faible dans une région et très fort dans l'autre. Paradoxalement, en essayant d'attirer le capital dans les régions les plus pauvres, les inégalités individuelles peuvent augmenter. Autrement dit: vous prenez aux pauvres des régions riches pour donner aux riches des régions pauvres. Un aspect important de ce débat repose sur la question des délocalisations.

Cette question est un peu moins au centre des débats politiques qu'il y a quelques années, mais peut revenir sur le devant de la scène à l'occasion d'une future délocalisation importante. Les économistes ont beaucoup insisté sur le fait que le phénomène de délocalisation vers l'étranger des emplois industriels français était, au niveau agrégé - je reviendrai sur ce terme -, assez faible. Il ne suffisait pas à expliquer le taux de chômage élevé en France. Pour résumer, les chiffrages expliquaient qu'entre $2 \%$ et $4 \%$ de la destruction d'emplois industriels étaient dus à des délocalisations vers l'étranger. Entre parenthèses, et même si l'on ne possède pas beaucoup de chiffres à ce sujet, il est probable que les délocalisations à l'intérieur même de la France sont plus importantes que celles effectuées vers l'étranger. Ces dernières ne constituent donc pas la bonne manière d'aborder le problème. En revanche, ce qui est vrai, c'est que ces délocalisations peuvent avoir une influence majeure sur quelques zones d'emploi spécifiques. Pour étayer cette remarque, il faut savoir que la France possède plus de 348 zones d'emploi. Parmi celles-ci, six ont subi $10 \%$ des destructions d'emplois industriels. C'est un chiffre effectivement assez concentré, mais il dépasse rarement cette barre des $10 \%$ ou $20 \%$ et la délocalisation ne peut donc pas être considérée comme la raison majeure de la destruction d'emplois. Le problème des dynamiques locales est donc posé. La géographie économique est soumise à beaucoup de phénomènes cumulatifs. Ainsi, si une entreprise part, ses clients et fournisseurs vont en pâtir, de même que les travailleurs. Ce processus de désindustrialisation et d'appauvrissement peut donc être, à un niveau plus microspatial que sur l'ensemble du territoire, déclenché par un phénomène cumulatif.

\section{Gagnants et perdants}

Ce problème de la destruction des emplois par la délocalisation est plus généralement recouvert par le débat sur les perdants de la mondialisation industrielle. Dans le cas présent, ces perdants sont à la fois l'individu et la région. C'est pourquoi se posent des questions d'équité mais aussi d'effica- 
cité. Car, et c'est le cas en France, s'il n'y a pas assez de compensation pour les perdants de la mondialisation, cela génère des réactions qui peuvent aller contre la mondialisation. Alors pourquoi compenser plus, ou mieux, la personne qui perd son emploi à cause de la mondialisation quand nous savons, par exemple, que le progrès technologique est également source de perte d'emploi? La meilleure réponse à cet argument, si l'on compare ces deux phénomènes, est de constater leurs différences. D'un côté, le choix de l'ouverture commerciale est un choix politique et, en partie, réversible. Le refus de la Constitution européenne de 2005 peut ainsi s'interpréter de cette manière. Lorsque les économistes affirment que cette ouverture crée des gagnants et des perdants mais que les gains des premiers sont supérieurs aux pertes des seconds, ils oublient le pendant de cette proposition. Idéalement, il faudrait taxer les profits de ces gagnants et les redistribuer aux perdants. Ce constat est important dans la mesure où ce choix de l'ouverture est politique et non subi. Historiquement, nombre de retours sur ces choix ont été effectués même s'ils deviennent de plus en plus difficiles de nos jours. Or, cette éventuelle redistribution des gains vers ceux qui peuvent perdre n'est jamais vraiment mise en œuvre, en particulier en Europe et en France. Visiblement, les pays scandinaves savent mieux le faire. Toujours est-il qu'il n'existe pas de programme spécifique pour dédommager les perdants de l'intégration commerciale.

Ainsi, l'élargissement de la communauté européenne aurait pu être mieux vécu s'il avait été précédé d'un programme prévoyant des compensations pour ceux qui pouvaient y perdre. Des programmes existent pourtant, tel le Trade Adjustment Act américain qui est très imparfait et incomplet. Ce type de programme pourrait cependant être envisagé en Europe avec la mise en place d'une aide forfaitaire pour ceux qui perdent leur emploi du fait d'une délocalisation, une autre aide spécifique à la reconversion, des diminutions d'impôts pour les territoires affectés ou bien encore l'obligation donnée aux entreprises qui délocalisent de payer des taxes aux collectivités locales qu'elles quittent. Plus généralement, et audelà de la dimension géographique des inégalités, la difficulté repose sur la compensation et la réinsertion des perdants dans le marché du travail. $\mathrm{Au}$ moment où la mondialisation commerciale nécessiterait plus de redistribution vers les individus et les territoires qui perdent pour stopper l'augmentation de ces inégalités, la mondialisation financière, la mobilité du capital et la concurrence fiscale qu'elle génère font qu'il est de plus en plus difficile de taxer pour redistribuer. C'est une question extrêmement importante concernant le débat sur la mondialisation.

Philippe Manière: Merci beaucoup pour cet éclairage passionnant sur les inégalités territoriales. Avant de donner la parole à David Thesmar, je voudrais vous poser quelques questions. Vous affirmiez redouter des inégalités entre États membres après la constitution du marché unique car la mobilité des travailleurs ne pouvait pas s'effectuer. Il est vrai qu'un travailleur limogé d'un chantier naval dans le sud du Portugal ne va pas retrouver un emploi au Danemark. Le constat est différent aux ÉtatsUnis où il est beaucoup moins traumatisant de bouger, et ce, même si le phénomène n'est pas massif. En Europe, vous l'avez souligné, nous avons 
surtout assisté à des déséquilibres territoriaux internes, ce qui n'était pas prévu. Alors pourquoi cet ajustement de la mobilité de la main-d'œuvre, qui ne paraît se heurter à aucun obstacle - si je perds mon travail à Charleville-Mézières, rien ne m'empêche de retrouver un emploi à Valence ou Cergy-Pontoise -, n'existe-t-il pas en France? En tout cas, pourquoi est-il moindre qu'au Royaume-Uni, en Espagne, en Italie ou en Allemagne?

Philippe Martin: Il n'y a pas de mobilité entre les pays européens et ce point est facile à comprendre. Et, à titre comparatif, cette mobilité à l'intérieur même des pays, est moindre que celle existant aux États-Unis. Cette dernière est, par exemple, beaucoup plus présente entre le Massachusetts et la Californie quand il y a une récession, qu'en France entre la Lorraine et le sud du pays. Cela peut s'expliquer par des phénomènes culturels et sociologiques sur lesquels je ne m'étendrai pas. Le constat vaut également pour l'Italie où les solidarités familiales peuvent limiter la mobilité entre les régions. La Grande-Bretagne, même si elle est moins touchée que la France, l'Italie ou l'Espagne, ne connait pas non plus une grande mobilité. Donc, au-delà des identités culturelles qui peuvent expliquer ce phénomène, ce dernier peut également s'appréhender par le biais des politiques publiques qui découlent du rôle de l'État providence et qui limitent clairement l'incitation à bouger. Il en va de même avec l'indemnisation chômage qui, si elle n'est pas une mauvaise chose, constitue un frein à aller chercher un travail très loin.

Philippe Manière: À rebours, puisqu'il n'est pas possible de compter sur l'État du Massachusetts, le départ en Californie s'impose.

Philippe Martin : La migration a un coût familial, social, etc. En France, la couverture sociale limite cette mobilité. D'autres politiques publiques ont également un impact sur la mobilité, comme l'absence de coopération entre les offices de HLM d'une ville à l'autre. De ce fait, l'habitant d'un HLM d'une ville trouvant un travail ailleurs perd le bénéfice de ce logement social. Ce n'est pas le logement social qui est à mettre en cause, mais la manière dont il est géré. Beaucoup de politiques publiques ont un effet sur la mobilité. Mais, quand bien même elle serait favorisée, des phénomènes culturels et sociologiques font qu'en Europe elle restera peu importante. Cela explique pourquoi aux États-Unis les travailleurs suivent, un peu passivement, la localisation des entreprises, tandis qu'en Europe, c'est l'inverse: les politiques publiques incitent les entreprises à se localiser là où les travailleurs veulent rester. Cet autre modèle a clairement des conséquences.

Philippe Manière: Parmi les autres aides, vous listez également l'accession à la propriété qui fixe les gens sur un territoire. Première provocation de ma part: vous avancez le fait qu'il faudrait plus de redistribution, compte tenu de l'accroissement des inégalités interterritoriales, et, en même temps, que l'Alsace et l'Île-de-France sont les deux seules régions où les revenus privés sont supérieurs aux revenus de transfert. Pourquoi 
n'est-ce pas le cas en région Rhône-Alpes, proche de la frontière? Mais surtout, l'Alsace et l'Île-de-France financent largement le reste du pays, ce qui prouve bien qu'existe déjà une redistribution massive entre les territoires surdotés en entreprises privées et ceux qui en ont peu. Il ne s'agit pas de prendre la défense du contribuable de ces deux régions, mais est-il bien raisonnable d'aller encore plus loin en ce sens? Dire «vivre et travailler dans son pays est un droit " - hommage à la CFDT! - ne revient-il pas à chasser les Franciliens à Londres?

Philippe Martin: De ce point de vue, en France, l'État providence a joué un rôle majeur - d'où le fait que les inégalités entre régions ne se sont pas traduites en termes de revenus. Les transferts sont très nombreux via l'impôt, l'indemnité chômage, etc. Le fait-on bien? La question n'est pas tant le montant de la redistribution entre les régions, mais la manière dont cela est fait: par exemple, donner des incitations aux entreprises, sous forme de subventions de capital, pour se relocaliser n'est peut-être pas la meilleure méthode. Je crois que des subventions au travail seraient, de ce point de vue, plus efficaces.

Philippe Manière: Le transfert des zones riches au bénéfice des zones pauvres rappelle le totem français de l'aménagement du territoire. Il n'est pas sans fondement, mais pourquoi taxer l'ouvrier de Poissy pour que le retraité installé dans le Perche puisse aller gratuitement poster ses cartes de vœux à Noël? Toute redistribution n’est pas forcément sociale.

Philippe Martin: C'est même pire que cela. Les entreprises implantées dans les régions pauvres sont subventionnées, mais les bénéfices en repartent.

Philippe Manière: Autre paradoxe: un jour, un patron fermait «dans la douleur " un site en Bretagne, dans une région mono-industrielle. Cela lui a créé des ennuis avec la presse, et la télévision commentait les événements tous les soirs. La conclusion qu'il en a tirée est de ne plus jamais ouvrir une usine dans un bassin déprimé: fermer une usine à Orsay ou à la Défense n'est pas un souci, car le personnel retrouve du travail et la télévision ne vient pas. Tandis qu'ouvrir une usine à Lannion, Colmar, Genlis en Côted'Or, où le taux de chômage est de $35 \%$, revient à rendre impossible, en cas de nécessité, l'éventualité de fermer cette entreprise. Les phénomènes cumulatifs, évoqués tout à l'heure, sont encore accrus par l'exposition médiatique: cela n'incite pas un employeur à s'installer en ces endroits, au regard des régions où il $\mathrm{y}$ a déjà de l'emploi. Comment rompre cette logique?

Philippe Martin: Cela joue dans les deux sens. En cas d'ouverture d'entreprise, une coopération entre les différents acteurs peut se produire, avec les syndicats, les élus locaux, etc. De ce fait, il n'est pas certain que l'exemple cité soit généralisable.

Notes

* Professeur d'économie à l'université de Paris I-Sorbonne. Voir ses travaux et ses articles. 1 